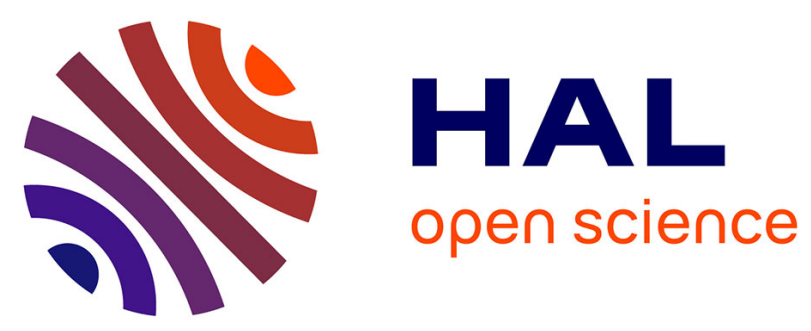

\title{
ETUDE MICROSTRUCTURALE DE L'AMORPHISATION SUPERFICIELLE D'UN ALLIAGE BASE FER, IRRADIE PAR LASER CO2 CONTINU
}

\author{
E. Gaffet, G. Deluze, G. Martin, Jean Pelletier
}

\section{To cite this version:}

E. Gaffet, G. Deluze, G. Martin, Jean Pelletier. ETUDE MICROSTRUCTURALE DE L'AMORPHISATION SUPERFICIELLE D'UN ALLIAGE BASE FER, IRRADIE PAR LASER CO2 CONTINU. Journal de Physique Colloques, 1987, 48 (C7), pp.C7-131-C7-134. 10.1051/jphyscol:1987723 . jpa-00227025

\section{HAL Id: jpa-00227025 https://hal.science/jpa-00227025}

Submitted on 1 Jan 1987

HAL is a multi-disciplinary open access archive for the deposit and dissemination of scientific research documents, whether they are published or not. The documents may come from teaching and research institutions in France or abroad, or from public or private research centers.
L'archive ouverte pluridisciplinaire HAL, est destinée au dépôt et à la diffusion de documents scientifiques de niveau recherche, publiés ou non, émanant des établissements d'enseignement et de recherche français ou étrangers, des laboratoires publics ou privés. 


\title{
ETUDE MICROSTRUCTURALE DE L'AMORPHISATION SUPERFICIELLE D'UN ALLIAGE BASE FER, IRRADIE PAR LASER $\mathrm{CO}_{2}$ CONTINU
}

\author{
E. GAFFET, G. DELUZE, G. MARTIN et J.M. PELLETIER* \\ CECM, CNRS, 15, Rue Georges Urbain, F-94407 Vitry-sur-Seine \\ Cedex, France \\ "CALFETMAT, Bâtiment 403, INSA-LYON, F-69621 Villeurbanne Cedex, \\ France
}

Résumé - Nous présentons une étude détaillée, par microscopie électronique, de la microstructure d'un alliage $\mathrm{Fe}-\mathrm{Cr}-\mathrm{P}-\mathrm{C}-\mathrm{Si}$, refondu superficiellement par laser. Des échantillons sont prélevés par ultramicrotomie, à diverses profondeurs dans la zone traitée. La structure obtenue lors de la solidification rapide est complexe et que partiellement amorphe: une solution très riche en métallozdes est observée entre les dendrites; cette solution se présente sous forme de lamelles à l'intérieur desquelles des zones amorphes sont détectées.

Abstract - A detailed study of the mtcrostructure of $\mathrm{CO}_{2}$ laser amorphtzation of a $\mathrm{Fe}-\mathrm{Cr}-\mathrm{P}-\mathrm{C}-\mathrm{Si}$ alloy is presented; electron micrographs of samples taken by ultramicrotomy at various depthes in the laser melted zone show that the amorphization is by no means uniform. A metalloid-rich phase is rejected in the interdendritic space and solidifies with a lamellar morphology. Amorphous phase may develop, under appropriated conditions, inside the lamellar areas.

\section{INTRODUCTION}

L'attrait de l'amorphisation superficielle d'une pièce métallique résulte des excellentes propriétés mécaniques et electrochimiques que possèdent certains verres métalliques, obtenus jusqu'à présent sous forme de rubans minces, notamment par la méthode classique de la trempe sur roue. Les traitements laser, de par leurs caractéristiques (forte densité de puissance, possibilités de refroidissement très rapide), permettent la réalisation d'une couche superficielle sur divers matériaux; deux cas ont été considérés :

- soit l'emploi de lasers pulsés de très faible durée de pulse ( de quelques nanosecondes, voire moins); la profondeur traitée ne dépasse alors pas quelques centaines de nanomètres $(1-6)$.

- soit l'emploi de lasers continus, essentiellement $\mathrm{CO}_{2}$; l'échantillon défile alors très rapidement sous le faisceau; les couches obtenues peuvent atteindre plusieurs dizaines de microns $(7-9)$.

C'est ce dernier cas que nous avons retenu. La caractérisation peut se faire:

- soit par diffraction des rayons X : la nature cristalline ou amorphe de la zone refondue peut être précisée, ainsi que la nature des phases formées.

- soit par microscopie électronique à transmission: cette méthode est plus délicate de mise en oeuvre, mais permet une étude plus fine des phénomènes (5). Cependant la localisation de la zone observée n'est pas précisée. Aussi avons-nous utilisé la technique de l'ultramicrotomie qui permet de réaliser des lames minces à diverses profondeurs, bien controlées, sous la surface de l'échantillon et donc de caractériser la structure obtenue par solidification rapide.

\section{METHODE EXPERIMENTALE}

La composition du matériau retenu est la suivante : Fe $74.5^{-} \mathrm{Cr}_{4} .3^{-} \mathrm{P}_{7.9-} \mathrm{C}_{11.2^{-}}$ Si2.1 ( $\%$ at.); les échantilions sont sous forme massive ( $\left.30 \times 30 \times 8 \mathrm{~mm}^{3}\right)$ et 
polis au diamant. Ils sont irradiés par faisceau laser continu avec une densité de pulssance de $8 \times 10^{5} \mathrm{~W} / \mathrm{cm}^{2}$. Un système toumant permet de déplacer la pièce sous le faisceau à des vitesses comprises entre 0.5 et $2 \mathrm{~m} / \mathrm{s}$; compte-tenu du diamètre de la zone irradiée (environ $0.8 \mathrm{~mm}$ ), les temps d'interaction varient donc entre 1.6 et $0.4 \mathrm{~ms}$. Pour obtenir une zone traitée de taille sufisante, c'est à dire de plusieurs millimètres de large, 12 passages successifs sont réalisés avec un décalage de 0.2 mm entre deux. Une protection gazeuse (argon) au niveau de la pièce permet d'éviter l'oxydation du matériau.

Les diagrammes de diffraction sont obtenus en utilisant la raie $K_{\alpha}$ du cuivre.

Les lames minces destinées à l'observation en microscopie electronique (microscope JEOL 2000FX) sont préparées par ultramicrotomie selon la méthode suivante :

- prélèvement à l'aide d'une scie diamantée d'une sorte d'allumette $\left(0.5 \times 0.5 \times 5 \mathrm{~mm}^{3}\right)$ dans la zone traitée par laser, perpendiculairement à la surface;

- après enrobage dans une résine epoxy des lames minces d'épatsseur voisine de 100nm sont découpées à l'aide d'un couteau diamanté, soit parallèlement soit perpendiculairement à la grande dimension de l'allumette. Cinq coupes successives sont regroupées sur la même grille et observées simultanément au microscope.

La microdiffraction et la microanalyse $X$ permettent de préciser les caractéristiques structurales et chimiques des diverses zones.

\section{RESULTATS EXPERIMENTAUX}

Les diagrammes de diffraction $X$ obtenus sur les divers échantillons sont présentés dans une étude parallèle $(10)$; lorsque la vitesse $v$ de défilement de la pièce sous le faisceau augmente, donc lorsque le temps d'interaction faisceau-matière diminue, nous observons une disparition progressive des pics de diffraction caractéristiques de l'état cristallin; pour $v=2 \mathrm{~m} / \mathrm{s}$ seule subsiste une large bosse analogue à celle obtenue sur les verres métalliques élaborés par trempe sur roue. Mais la couche traitée est-elle amorphe? Les observations par microscopie électronique révèlent la pésence de deux zones :

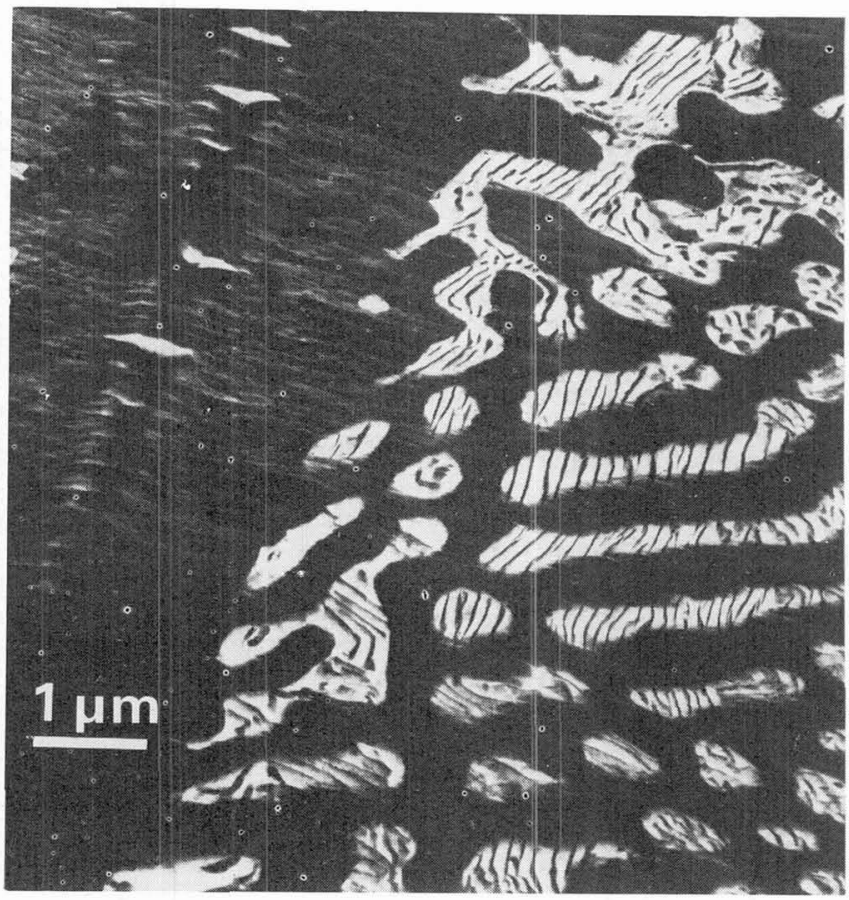

Fig. 1 - Image en champ clair d'une coupe parallèle à $1 a$ surface de l'échantilion $(v=1.5 \mathrm{~m} / \mathrm{s})$.

Bright field image; cut parallel to the free surface $(v=1.5 \mathrm{~m} / \mathrm{s})$. 
- près de la surface une zone peu contrastée; celle-ci est homogène et aucune dendrite ou cellule n'est détectée. Cependant la microdiffraction indique que si certaines parties sont amorphes, quelques hétérogénéités cristalinnes existent. - en dessous, c'est à dire pour des profondeurs supérieures à $0.5 \mu \mathrm{m}$, nous observons très nettement une structure de type dendritique (Fig.1); pour v= $1.25 \mathrm{~m} / \mathrm{s}$ l'espacement interdendritique est voisin de $2 \mu \mathrm{m}$; il existe une très grande diversité de morphologies, même à une profondeur donnée. Les dendrites sont cristallines et constituées essentiellement d'éléments métalliques (tableau I) (en noir sur la micrographie). Les métalloides $\mathrm{P}$ et $\mathrm{Si}$ (le carbone ne peut pas être analysé par EDX) sont fortement ségrégés dans les zones interdendritiques; celles-ci se présentent sous forme de lamelles alternées: les lamelles noires sont également peu chargées en métalloides et leur structure est cristalline, ajors que dans les zones claires la concentration en phosphore et en silicium est très élevée; seules ces dernières zones contiennent une phase amorphe. La présence de ces lamelles ne peut en aucun cas résulter d'un artefact dû à la méthode de préparation, puisqu'elles sont aussi bien parallèles que perpendiculaires à la direction de propagation du couteau. Dans certains cas nous observons des zones interdendritiques de très faible dimension $(\approx 100 \mathrm{~nm}$ ); leur structure est amorphe et elles contiennent une très forte proportion de phosphore.

\begin{tabular}{cccccc}
\hline & & $\begin{array}{c}\text { espace } \\
\text { dendrites } \\
\text { interdendritique } \\
\text { (valeur moyenne) }\end{array}$ & $\begin{array}{c}\text { lamelles } \\
\text { cristallines }\end{array}$ & $\begin{array}{c}\text { lamelles } \\
\text { amorphes }\end{array}$ & $\begin{array}{c}\text { espaces } \\
\text { interdendritique } \\
\text { amorphes }\end{array}$ \\
\hline $\mathrm{Fe}$ & 85.9 & 84.7 & 87.3 & 78.6 & 70.2 \\
\hline $\mathrm{Cr}$ & 12.2 & 9.3 & 8 & 6.5 & 4.8 \\
\hline $\mathrm{Si}$ & 0.3 & 4.2 & 3.3 & 12.2 & 12.9 \\
\hline $\mathrm{P}$ & - & - & 1.5 & 2.6 & 8.0 \\
\hline
\end{tabular}

Tableau I : Concentration en différents éléments suivant les zones (EDX microanalysis)

La variation du temps $\tau$ d'interaction laser-matériau ne modifie pas la nature cristailine des dendrites et le volume occupé par les zones interdendritiques reste pratiquement constant; par contre la proportion de phase amorphe evolue: lorsque diminue le volume des zones riches en métalloides augment; pour des vitesses $v$ inférieures à $1.25 \mathrm{~m} / \mathrm{s}$ il $\mathrm{n}$ 'y a pas toujours présence de phase amorphe dans les zones interdendritiques, alors que pour $v \geqslant 1.25 \mathrm{~m} / \mathrm{s}$ cette présence est systématique; dans cette phase amorphe la distance entre les plus proches voisins est de l'ordre de $1.95 \AA$, ce qui est une valeur typique des alliages base fer non-cristallins.

\section{DISCUSSION}

La formation d'un verre métallique dépend très fortement de la vitesse de refroidissement et des possilitiés de croissance cristalline. Lors de la refusion par laser et de la solidification qui suit le liquide est en contact direct avec le substrat resté froid. le matériau étant le même, le coefficient de transfert 
thermique sera donc très élevé, ce qui autorise des vitesses de trempe très importantes; ceci a un effet bénéfique pour la vitrification. En revanche la nucléation de la phase cristalline lors de la solidification de la couche liquide est favorisée par la présence du substrat cristallin, de même composition chimique et en contact direct. Cet effet constitue un obstacle à la création de phase amorphe. Au vu des observations micrographiques nous proposons le mécanisme suivant pour la solidification : des dendrites commencent par se solidifier; cette solidification s'accompagne du phénomène classique de rejet solutal, conséquence d'un sous-refroidissement constitutionnel $(11,12)$; le liquide interdendritique se trouve ainsi enrichi en éléments chimiques favorisant l'amorphisation des alliages base fer, à savoir $\mathrm{P}$ et $\mathrm{Si}$ (et eventuellement C). Seules ces zones riches en métalioides peuvent se solidifier à l'état amorphe. Un mécanisme de même nature peut être envisagé pour la formation des lamelles à l'intérieur des zones interdendritiques. La diminution de la distance entre les dendrites lorsque le temps d'interaction diminue s'explique par l'augmentation de la vitesse de trempe consécutive à cette variation.

Juste en dessous de la surface plusieurs facteurs favorisent l'amorphisation :

- la possibilité d'échange thermique avec le milieu extérieur contribue à une augmentation de la vitesse de refroidissement;

- la phase liquide a vu sa teneur en métalioídes progressivement augmentée;

D'où la possibilité d'une solidification planaire avec formation d'une phase relativement homogène. Les quelques cristallites que I'on peut y détecter résultent peut être du recuit provoqué par le traitement laser multipasses (recouvrement des zones traitées).

\section{CONCLUSION}

La technique d'observation en microsopie électronique par transmission de lames minces préparées par ultramicrotomle permet d'analyser de façon très fine la structure qui résulte de la solidification rapide consécutive au trattement laser. Dans 1 'alliage $\mathrm{Fe}-\mathrm{Cr}-\mathrm{P}-\mathrm{C}-\mathrm{Si}$ la formation de dendrites primaires cristallines s'accompagne d'un rejet solutal important; la création de zones ainsi fortement enrichies en métallozdes permet la création de zones amorphes, dont la proportion augmente avec la vitesse de trempe, par le biais d'une diminution du temps d'interaction laser-matériau.

\section{REFERENCES}

11/ Fuxi,G., Baorong,S., Hao,W.,J. Non Cryst. Sol.,50 (1983) 201.

/2/ Lin,C.J., Spaepen,F., Turnbu11,D., J. Non Cryst. So1.,61 \& 62 (1984) 767.

13/ Blatter, A., Von Allmen,M., Phys. Rev. Letters, 54 (1985) 2103.

14/ AIden,D.A., Massalski,T.B., Lowndes,D.H., Kaufmann,E.N., Scripta Metall.,19 (1985) 67 .

15/ Woychik,C.G., Lowndes,D.H., Massalski,T.B., Acta Metall., 33 (1985) 1861.et 1873.

16/ Whittle,G.L., Calka,A., Radlinski,A.P., Luther-Davies,B., J. Magn. Magn. Mat., 50 (1985) 278 .

/7/ Becker,R., Sepold,G., Scripta Metall., 14 (1980) 1283.

18/ Bergmann,H.W., Mordike,B.L., Z. Metallkde, 71 (1980) 658.

19/ Yoshioka,H., Asami,K., Hashimoto,K., Scripta Metall., 18 (1984) 1215.

/10/ Pelletier,J.M., Pergue,D., cette conférence.

111 Elliot,R., dans "Eutectic solidification processing", Butterworths Monographs in Materials, Butterworths and Co Ltd (1983) 55.

$112 /$ Porter,D.A., Easterling,K.E., dans "Phase Transformations in Metals and Alloys", Van Nostrand Reinhold Co (1981) 208. 